\title{
The ventral route to intracranial aneurysm: from the origin towards modern transsphenoidal surgery. An historical review and current perspective*
}

\author{
Alberto Di Somma', Matteo de Notaris ${ }^{2,5}$, Joaquim Enseñat ${ }^{3}$, Isam Alobid ${ }^{4}$, \\ Manuel Bernal-Sprekelsen ${ }^{4}$, Luigi M. Cavallo', Alberto Prats-Galino5, Paolo \\ Cappabianca' \\ ' Department of Neurosciences, Reproductive and Odontostomatological Sciences, Division of Neurosurgery, \\ Università degli Studi di Napoli Federico II, Naples, Italy \\ 2 Department of Neuroscience, Division of Neurosurgery, "G. Rummo" Hospital, Benevento, Italy \\ ${ }^{3}$ Division of Neurosurgery, Hospital Clinic de Barcelona, Faculty of Medicine, Universitat de Barcelona, Barcelona, Spain \\ ${ }^{4}$ Department of Otorhinolaryngology, Rhinology Unit, Hospital Clinic de Barcelona, Faculty of Medicine, Universitat de Barcelona, \\ Barcelona, Spain \\ ${ }^{5}$ Laboratory of Surgical Neuroanatomy (LSNA), Faculty of Medicine, Universitat de Barcelona, Barcelona, Spain
}

Rhinology 52: 197-207, 2014 DOI:10.4193/Rhino13.165 *Received for publication: October 3, 2013 Accepted: December 3, 2013

\begin{abstract}
Objective: A review of the main studies that have explored the use of the ventral pathway for treatment of intracranial aneurys$\mathrm{ms}$, including the recent reported extended transsphenoidal approaches.

Methods: A comprehensive literature review was performed using the PudMed database. We recovered 48 cases of cerebral aneurysms, approached via the transcervical-transclival, transoral-transclival, transfacial-transclival ventral pathways and the extended transsphenoidal route. The overall rates of complications and surgical success were evaluated and compared for both traditional ventral and transsphenoidal approaches.
\end{abstract}

Results: For traditional routes, the overall complications and surgical success rates were $74 \%(26 / 35)$ and $87 \%$ (13/15), respectively. For extended transsphenoidal approaches were $44 \%$ (4/9) and $78 \%(7 / 9)$, respectively.

Conclusion: Our paper is a reconnaissance of what has been done via "the anterior route" and a notification of the existence of this "surgical window". Present and future of cerebral aneurysm treatment is represented by the endovascular technique. A few selected cases in specialized centers, where transsphenoidal approaches with the aid of the endoscope are routinely performed, may be treated with such techniques alone or in combination with other different procedures. Further studies in large numbers of patients will be required to validate the full benefit of this approach.

Key words: cerebral aneurysms, endoscopic endonasal approaches, minimally invasive surgery, skull base surgery

\section{Introduction}

The ventral route for intracranial aneurysms was first employed in late sixties to clip a basilar artery aneurysm via a transoraltransclival approach ${ }^{(1)}$ and it was not a coincidence that such a "peculiar" pathway for vascular surgery was successfully applied for a posterior circulation aneurysm, namely a proximal basilar artery aneurysm. The posterior circulation has historically been viewed as an area that presents a significant access challenge, because of the operative technical difficulties and the potential surgical morbidity. First of all, it has to be reminded that in the period between 1930 and 1960, surgical treatment of posterior circulation aneurysms was only possible by indirect trapping 
or parent vessel ligation and neurosurgeons were attempting direct surgical clipping of vertebrobasilar aneurysms via a transcranial route only in early sixties: Drake from Canada published his initial experience with four ruptured basilar bifurcation aneurysms in $1961^{(2)}$ and Jamieson from Australia reported 19 surgical cases in $1964^{(3)}$. He commented that "it is clear that the basilar bifurcation is no place for the faint of heart".

More recently, many authors ${ }^{(4-6)}$ stressed the concept that surgical access to vascular lesions behind the lower third of the clivus could be difficult by any route. However, it is possible to reach such complex anatomical areas from both the cerebellopontine angles and the transtentorial-suboccipital routes, but those aneurysms could be obscured from a vital neurovascular structure, i.e. the ventral exit of the glossopharyngeal, vagus, accessory, hypoglossal cranial nerves and from the vertebral artery (VA), posterior cerebral artery (P1 segment) and tonsillomedullary, lateralmedullary, and anteriormedullary segments of the posterior inferior cerebellar artery (PICA) ${ }^{(7)}$.

For such reason, several authors, over the past 50 years, began to plan alternative routes to get access to aneurysms arising from the midline posterior circulation. As a matter of fact, the ventral pathway was considered a reasonable alternative, because it permits direct access to the surgical field with exposure and dissection of the aneurysm from the surrounding neurovascular tissue, obviating brain retraction and obtaining an early and safe proximal and distal vascular control ${ }^{(1,8-13)}$.

More recently, with the advent of transsphenoidal surgery and, in particular, with the rapid development of the endoscopic endonasal technique, the interest in extended transsphenoidal approaches has been renewed for many pathological entities ${ }^{(14,15)}$. Particularly, the wider and panoramic view offered by the endoscope increased the versatility of the transsphenoidal approach and allowed it to be expanded to different parts of the skull base ${ }^{(16)}$. Indeed, a variety of vascular lesions, mainly posterior circulation aneurysm, have started to be approached through the transsphenoidal route, pioneered by the innovative works presented by Kassam et al. in 2006 and $2007^{(17,18)}$.

The purpose of the present contribution is to review the ventral approaches to intracranial aneurysms and to discuss its surgical results and complications over the years.

\section{Materials and methods}

A comprehensive literature review of manuscripts was performed as shown below.

\section{Literature analysis}

The search was conducted in the PubMed database using the following keywords (individually or in association): "transoral", "transcervical", "transclival", "transfacial", "transsphenoidal", "endonasal", "endoscopic" and "cerebral aneurysm". The date of the latest search was February 2013.
Selection criteria

All clinical manuscripts that approached aneurysms via the transcervical-transclival, transoral-transclival, transfacial-transclival or transsphenoidal routes were analyzed as well as their references.

\section{Data collection}

Manuscript selection was performed by two authors that independently reviewed the articles for inclusion or exclusion. No disagreements were found.

\section{Data analysis}

To calculate the overall complication rate, cases in which the authors described at least one complication, namely CSF leak, meningitis, cranial nerve injury, dysphagia, retropharyngeal mass (for example CSF collection in the retropharyngeal space), hydrocephalus, slip-out of the clip, cardiac arrest, pharyngeal wound infection, subarachnoid hemorrhage, vasospasm, mild postoperative confusion, deep venous thrombosis and death, were used. To determine the success or insuccess of the surgical performance, by means of postoperative computerized tomography (CT) or magnetic resonance (MR) or angiography, the following neuroradiological and clinical criteria have been evaluated including obliteration of the aneurysm sac, proper position of the clip and patency of the parent arteries.

Overall complication and surgical success rates were calculated for papers providing all required data.

\section{Historical background of the ventral approaches: complications, surgical results and reconstruction techniques}

Pioneering work: the transoral approach

In 1966, Sano et al. ${ }^{(1)}$ reported the first successful case of transoral-transclival coating and wrapping of an aneurysm of the proximal basilar artery, located at the lower third of the clivus, i.e. the so-called "no man's land". The authors stressed the advantages of this route in permitting dissection of the aneurysm under direct vision control without any retraction of the brain or the cranial nerves. Their patient did not report cerebrospinal fluid (CSF) leak and/or meningitis and recovered well. This pioneering report encouraged other authors to use the ventral approach in selected patients (Table 1 and 2, Figure 1).

\section{First transcervical-transclival approaches}

As previously described ${ }^{(8)}$, the transcervical-transclival approach is used to access lesions arising or extending into the craniovertebral junction, the lower part of the clivus up to C1. A transverse cervical incision, from the midline to the mastoid tip is performed. After dissected the underlying tissues, the clival bone can be visualized and partially removed. A midline dural incision is then made to reach the surgical target (basilar artery 


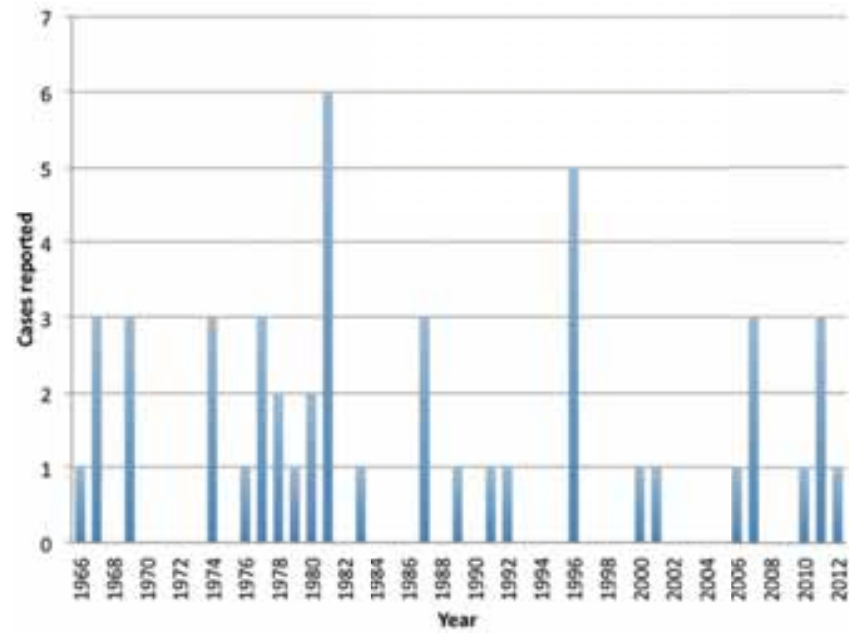

Figure 1. Graph showing 48 cases of cerebral aneurysms reported in the PudMed database and treated via the ventral route, from 1966 to 2012 (blue bars).

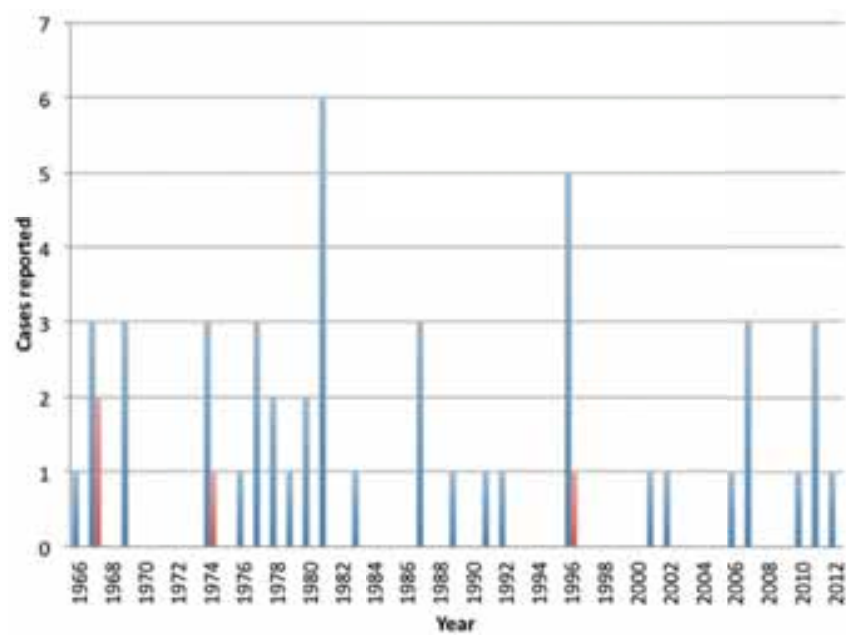

Figure 2. Graph showing the cases of aneurysms approached via the ventral route (blue bars) and cranial nerve lesion (red bars) as complication after surgery. In 1967 and 1974, the cases of cranial nerve injury were associated with transcervical-transclival approach, while the case reported in 1996 was related to trasfacial-transclival approach. or vertebral artery aneurysm).

Between 1967 and 1976, many authors reported cases of posterior brain circulation aneurysms treated via the transcervicaltransclival route ${ }^{(8)}$. In his "Microsurgery applied to Neurosurgery" monograph, Yaşargil (11) described two unsuccessful ligations of posterior circulation aneurysms attacked via the transcervicaltransclival route resulting in death of both patients. The most common postoperative complications were cranial nerve injury (hypoglossal nerve, XII; Figure 2), CSF leak and pharyngeal stenosis. In particular, Wissinger et al. ${ }^{(10)}$ and Chou et al. ${ }^{(13)}$ reported two cases of transection of the hypoglossal nerve and subsequent palsy. In those years, the skull base reconstruction technique was limited to free flaps from the pharyngeal mucosa and CSF leaks were present in the majority of cases.

Revival of the transoral-transclival approach In the following years, the transcervical route to get access to the clival region was gradually abandoned and many authors began to manage aneurysms of the posterior circulation via the transoral-transclival route.

As described ${ }^{(19)}$, the transoral-transclival approach allowed to reach the surgical target after a midline hard and soft palatal split and mobilizing each hemi-maxillae laterally (following or not a standard Le Fort I maxillotomy).

Surgical results and outcomes were in most of cases acceptable ${ }^{(12,20-28)}$. Nevertheless, Drake et al. ${ }^{(29)}$, Hashi et al. ${ }^{\left({ }^{(3)}\right.}$, Saito et al. ${ }^{(9)}$, Matricali et al. ${ }^{(31)}$, and Hayakawa et al. ${ }^{(32-34)}$ reported 5 casualties related to surgery but the most common complications were CSF leak (Figure 3) and meningitis. However, the most dangerous event was the possibility that the clip could move away from the neck of the aneurysm. Indeed, in two cases, Saito et al. ${ }^{(9)}$ and Litvak et al. ${ }^{(25)}$, showed that the head of the clip was larger than the bone window in the clivus and, for this reason, the clip slipped out from the aneurysm neck, causing death of the patient in one case. Concerning the reconstruction technique, in 1979, Yamaura et al. ${ }^{(23)}$ published a notable work describing the

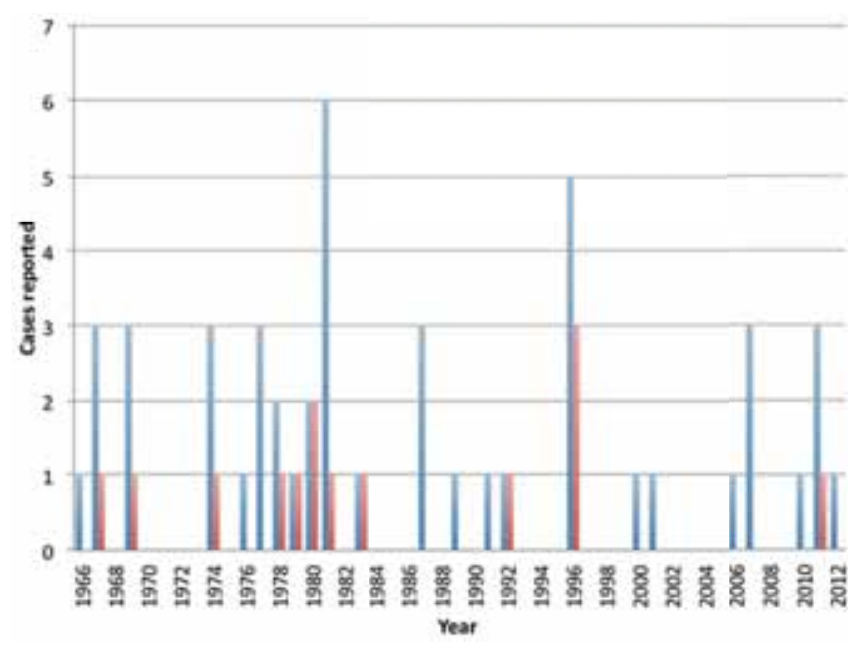

Figure 3. Graph showing the cases of aneurysms approached via the ventral route (blue bars) and cerebrospinal fluid (CSF) leak (red bars) as complication after surgery. Between 1966 and 1996, the cases of ce-rebrospinal fluid leak were related to transcervical-transclival, transoral-transclival and transfacial-transclival approaches, while the case reported in 2011 was associated with the endoscopic endonasal transclival approach. 

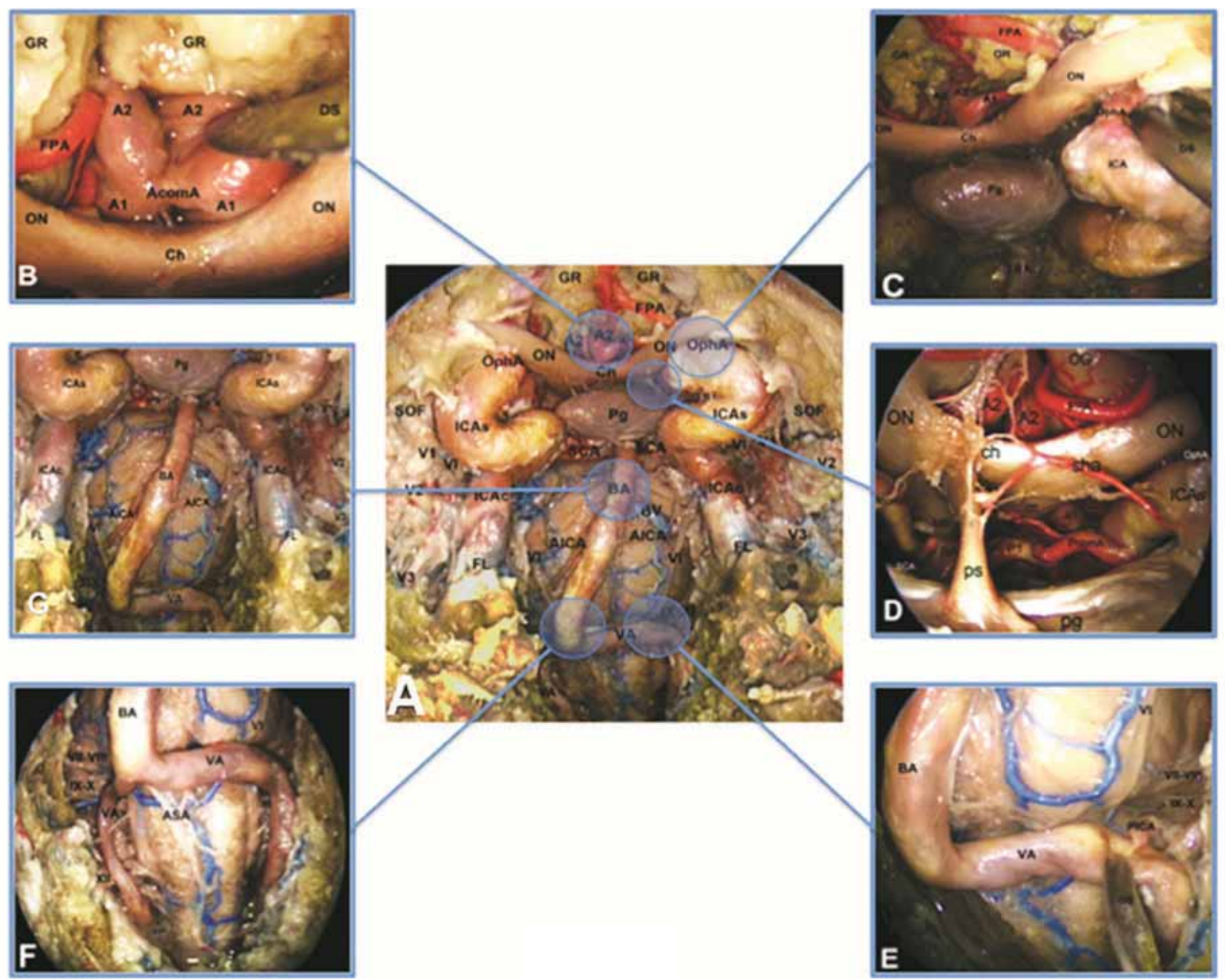

Figure 4. Anatomical picture: Dissection at the Laboratory of Surgical NeuroAnatomy of Barcelona, Spain. Vascular areas already reached via extended endoscopic endonasal approaches. Panoramic image (A) showing the main anterior and posterior circulation vessels exposed via extended endoscopic endonasal route. Close-up view of the suprasellar area with (B) the anterior communicating artery complex, (C) the ophthalmic artery and (D) the superior hypophyseal artery; detailed images $(E)$ of the posterior inferior cerebellar artery arising from the vertebral artery, (F) the origin of the basilar artery and (G) the basilar trunk; schematic drawing $(\mathrm{H})$ showing the entire cerebral circulation as seen through the ventral endoscopic view. $\mathrm{Ch}=$ optic chiasm; $\mathrm{ON}=$ optic nerve; $\mathrm{Pg}$ = pituitary gland; $\mathrm{GR}=$ gyrus rectus; $\mathrm{FPA}=$ frontopolar artery; $\mathrm{A} 1=$ pre-communicating tract of the anterior cerebral artery; $\mathrm{A} 2$ = post-communicating tract of the anterior cerebral artery; $A$ com $A$ $($ Acom $)=$ anterior communicating artery; OphA $=$ ophthalmic artery; ICA $=$ internal carotid artery; ICAs = sellar segment of the internal carotid artery; ICAC = clival segment of the internal carotid artery; FL = foramen lacerum; SOF = superior orbital fissure; $\mathrm{Vl}=$ abducent nerve; $\mathrm{GG}=$ gasserian ganglion; $\mathrm{V} 1$ = ophtalmic branch of the trigeminal nerve; $\mathrm{V} 2$ $=$ maxillary branch of the trigeminal nerve; $\mathrm{V} 3=$ mandibular branch of the trigeminal nerve; VII-VIII = acoustic-facial nerve bundle; IX = glossopharyngeal nerve; $\mathrm{X}=$ vagus nerve; $\mathrm{XII}$, hypoglossal nerve; sha =



superior hypophyseal artery; PcomA (Pcom), posterior communicating artery; $\mathrm{PCA}=$ posterior cerebral artery; $\mathrm{P} 2$ = post-communicating tract of the posterior cerebral artery; $\mathrm{SCA}=$ superior cerebellar artery; $\mathrm{BA}=$ basilar artery; $\mathrm{BV}=$ basilar venous plexus; $\mathrm{ASA}=$ anterior spinal artery; $\mathrm{VA}=$ vertebral artery; $\mathrm{AICA}=$ anteriorinferior cerebellar artery; $\mathrm{PICA}=$ posteriorinferior cerebellar artery; DS = dissector. 


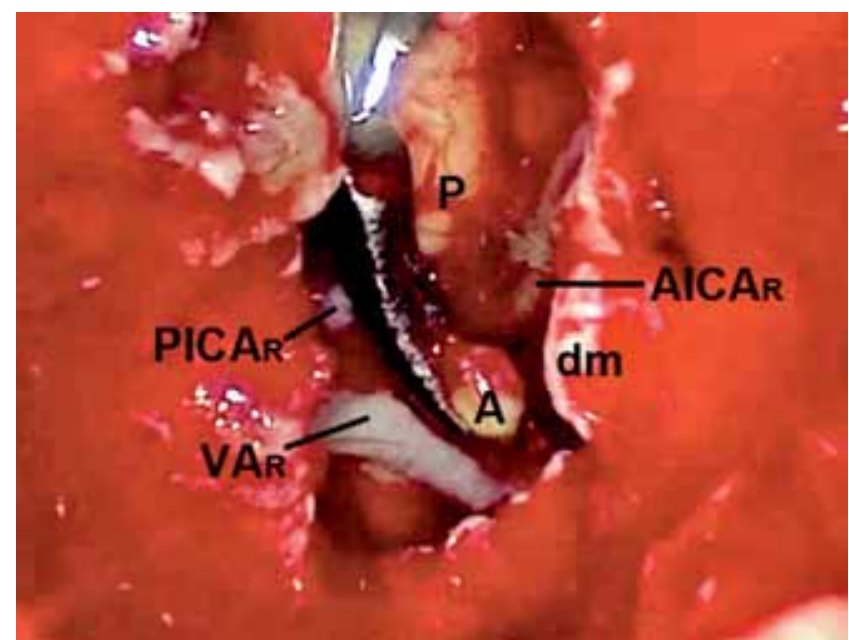

Figure 5. Endoscopic endonasal inferior transclival view of a vertebral artery-posterior inferior cerebellar artery aneurysm clipping. $A=$ aneurysm; $\mathrm{AICAr}=$ right anterior inferior cerebellar artery; $\mathrm{dm}=$ dura mater; $\mathrm{P}=$ perforator arteries; $\mathrm{PICAr}=$ right posterior inferior cerebellar artery; $\mathrm{VAr}=$ right vertebral artery.

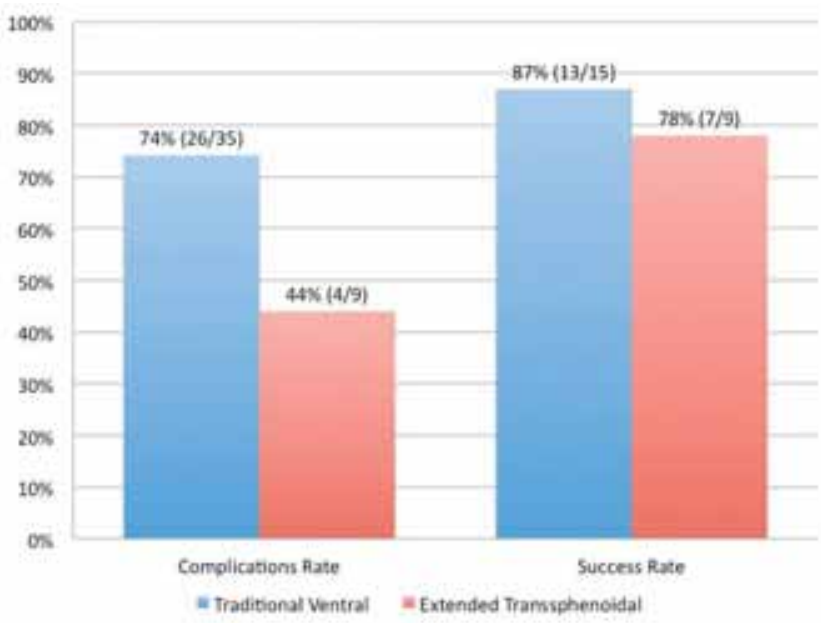

Figure 6. Graph showing the complications rate and the success rate of traditional ventral (blue bars) and extended transsphenoidal approaches (red bars), performed in order to treat cerebral aneurysms. first report of employment of a vascularized flap for transclivaltransoral approach; indeed, after the clipping of a basilar artery aneurysm, the authors repaired the postoperative CSF fistula using a rotation flap of the nasal septum mucosa, which was exploited to cover the skull base defect.

In the next years, Archer et al. ${ }^{(35)}$, de Los Reyes et al. ${ }^{(36)}$, and Crockard et al. ${ }^{(37,38)}$ described a modification of the transoraltransclival route involving a Le Fort I osteotomy (maxillotomy) rather than splitting the soft and hard palates, offering a much improved view of the clivus. The authors achieved a reduced rate of CSF fistula thanks to the improved exposure provided by the Le Fort I osteotomy. Another notable case report was described by Crockard et al. ${ }^{(19)}$. In their paper, the authors showed the occlusion of an aneurysm with a curved variangle McFadden clip enforced using a rotating pistol-grip aneurysm clip applier that was especially developed for transoral vascular surgery.

\section{Transfacial-transclival approach}

In 1996, Ogilvy et al. ${ }^{(39)}$ described five patients with midposterior circulation aneurysms treated through a transfacialtransclival approach.

A lateral rhinotomy incision is usually made from the glabella around the lateral alar margin. After osteotomy of nasal bones and disarticulation of the septal cartilage from the ethmoid, the nose can be reflected laterally. Further dissection of the retropharyngeal mucosa permitted to perform the bone removal on the clivus. Afterwards, the dura can be opened to reach the midline target ${ }^{(39)}$.

For reconstruction, the authors used a multilayer closure including free fat grafts, septal bone, cartilage, fascia lata, fibrin glue, and an overlying split-thickness skin graft, as well as perioperative drainage with lumbar drains and ventriculostomies. Despite these measures, three of their patients developed transient CSF leaks after nasal packs removal.

In the years following, due to frequent complications, mainly meningitis for recurrent CSF leak, the transcervical, transoral and transfacial approaches for the treatment of cerebral aneurysms were gradually discarded.

Minimally invasive approaches: transsphenoidal approaches and extended endoscopic endonasal surgery The evolution of the transsphenoidal technique over the last decade, resulting largely from better instrumentation, surgical navigation and, perhaps most importantly, endoscopy, has lead to the extension of the approach beyond treating pituitary adenomas to the management of different skull base lesions. Indeed, during the early nineties, the endoscopic endonasal approach has been progressively accepted by neurosurgeons, and in many centers throughout the world, this route has been used following the same indications of conventional microsurgical technique. Currently, a variety of anatomical and clinical studies have demonstrated the possibility to reach different areas of the skull base ${ }^{(40-43)}$.

These apporaches permitted to reach the midline target passing through the sphenoid sinus, by means of a microscope or an endoscope.

For vascular surgery, extended endoscopic endonasal approaches provide excellent exposure of important anterior and posterior circulation arteries. For this reason, up to now, a total 
Table 1. Clinical and operative characteristics in 39 reported patients with intracranial aneurysms treated via the "traditional" ventral routes (transcervical-transclival, transoral-transclival, transfacial-transclival), literature review.

\begin{tabular}{|c|c|c|c|c|c|c|}
\hline Authors \& Year & $\begin{array}{l}\text { Aneurysm } \\
\text { location \& } \\
\text { size }(\mathbf{m m})\end{array}$ & $\begin{array}{l}\text { Clinical } \\
\text { presentation }\end{array}$ & Approach & $\begin{array}{l}\text { Post-operative } \\
\text { imaging }\end{array}$ & Complications & Outcome \\
\hline $\begin{array}{l}\text { Sano et al., } \\
1966\end{array}$ & $\mathrm{BA}(\mathrm{NA})$ & NA & $\begin{array}{l}\text { Transoral- } \\
\text { transclival }\end{array}$ & NA & None & Full recovery \\
\hline $\begin{array}{l}\text { Fox et al., } \\
1967\end{array}$ & VA (15) & $\begin{array}{l}\text { Headache, hyperactive } \\
\text { OTR, ataxia }\end{array}$ & $\begin{array}{l}\text { Transcervical- } \\
\text { transclival }\end{array}$ & $\begin{array}{l}\text { Aneurysm } \\
\text { obliterated }\end{array}$ & $\begin{array}{l}\text { CSF leak, dysphagia, re- } \\
\text { tropharyngeal mass (CSF } \\
\text { collection), hydrocephalus }\end{array}$ & $\begin{array}{l}\text { Cranial Nerve } \\
\text { Lesion }\end{array}$ \\
\hline \multirow[t]{2}{*}{$\begin{array}{l}\text { Wissinger et al., } \\
1967\end{array}$} & VA-BA (NA) & $\mathrm{SAH}$ & $\begin{array}{l}\text { Transcervical- } \\
\text { transclival }\end{array}$ & NA & None & Full recovery \\
\hline & $\mathrm{BA}(\mathrm{NA})$ & $\mathrm{SAH}$ & $\begin{array}{l}\text { Transcervical- } \\
\text { transclival }\end{array}$ & $\begin{array}{l}\text { Aneurysm } \\
\text { obliterated }\end{array}$ & Hypoglossal nerve injury & $\begin{array}{l}\text { Cranial Nerve } \\
\text { Lesion }\end{array}$ \\
\hline $\begin{array}{l}\text { Drake et al., } \\
1969\end{array}$ & VA (NA) & NA & $\begin{array}{l}\text { Transoral- } \\
\text { transclival }\end{array}$ & NA & Clip extrusion & Full recovery \\
\hline \multirow[t]{2}{*}{$\begin{array}{l}\text { Yasargil, } \\
1969\end{array}$} & VA-BA (NA) & NA & $\begin{array}{l}\text { Transcervical- } \\
\text { transclival }\end{array}$ & NA & $\begin{array}{l}\text { Hemiparesis, CSF leak? } \\
\text { ( } 1 \text { pz had CSF leak) }\end{array}$ & Death \\
\hline & $\mathrm{BA}(\mathrm{NA})$ & NA & $\begin{array}{l}\text { Transcervical- } \\
\text { transclival }\end{array}$ & NA & $\begin{array}{l}\text { Hydrocephalus, } \\
\text { tetraplegia, CSF leak? }\end{array}$ & Death \\
\hline \multirow[t]{3}{*}{$\begin{array}{l}\text { Chou et al., } \\
1974\end{array}$} & $\mathrm{BA}(10)$ & $\begin{array}{l}\text { HH grade II, headache, } \\
\text { transitory loss of vision, } \\
\text { coma }\end{array}$ & $\begin{array}{l}\text { Transcervical- } \\
\text { transclival }\end{array}$ & NA & $\begin{array}{l}\text { Hypoglossal nerve injury, } \\
\text { cardiac arrest }\end{array}$ & $\begin{array}{l}\text { Cranial Nerve } \\
\text { Lesion }\end{array}$ \\
\hline & $\mathrm{BA}(9)$ & $\begin{array}{l}\text { HH grade II, headache, } \\
\text { loss of consciousness }\end{array}$ & $\begin{array}{l}\text { Transcervical- } \\
\text { transclival }\end{array}$ & NA & $\begin{array}{l}\text { Vasospasm, } \\
\text { retropharyngeal mass }\end{array}$ & Full recovery \\
\hline & $\mathrm{BA}(7)$ & $\begin{array}{l}\text { HH grade II, seizure, } \\
\text { pulmonary edema }\end{array}$ & $\begin{array}{l}\text { Transcervical- } \\
\text { transclival }\end{array}$ & NA & Vasospasm, CSF leak & Full recovery \\
\hline $\begin{array}{l}\text { Hashi et al., } \\
1976\end{array}$ & VA (2) & $\begin{array}{l}\text { Bronchial asthma, } \\
\text { dysphagia, dysphonia }\end{array}$ & $\begin{array}{l}\text { Transoral- } \\
\text { transclival }\end{array}$ & NA & Meningitis & Death \\
\hline $\begin{array}{l}\text { Takeuchi et al., } \\
1977\end{array}$ & VA (NA) & NA & $\begin{array}{l}\text { Transoral- } \\
\text { transclival }\end{array}$ & NA & Meningitis, hydrocephalus & Full recovery \\
\hline \multirow[t]{2}{*}{$\begin{array}{l}\text { Laine et al., } \\
1977\end{array}$} & $\mathrm{AICA}(\mathrm{NA})$ & NA & $\begin{array}{l}\text { Transoral- } \\
\text { transclival }\end{array}$ & NA & NA & NA \\
\hline & PICA (NA) & NA & $\begin{array}{l}\text { Transoral- } \\
\text { transclival }\end{array}$ & NA & NA & NA \\
\hline $\begin{array}{l}\text { Drake et al., } \\
1978\end{array}$ & VA (NA) & NA & $\begin{array}{l}\text { Transoral- } \\
\text { transclival }\end{array}$ & NA & Meningitis, CSF leak & Death \\
\hline $\begin{array}{l}\text { Haselden et al., } \\
1978\end{array}$ & $\mathrm{BA}(\mathrm{NA})$ & NA & $\begin{array}{l}\text { Transoral- } \\
\text { transclival }\end{array}$ & NA & NA & Full recovery \\
\hline $\begin{array}{l}\text { Yamaura et al., } \\
1979\end{array}$ & $\mathrm{BA}(\mathrm{NA})$ & NA & $\begin{array}{l}\text { Transoral- } \\
\text { transclival }\end{array}$ & NA & CSF leak & Full recovery \\
\hline \multirow[t]{2}{*}{$\begin{array}{l}\text { Saito et al., } \\
1980\end{array}$} & VA-BA (NA) & $\begin{array}{l}\text { SAH, loss of conscious- } \\
\text { ness, dysdiadochokinesis }\end{array}$ & $\begin{array}{l}\text { Transoral- } \\
\text { transclival }\end{array}$ & $\begin{array}{l}\text { Aneurysm } \\
\text { obliterated }\end{array}$ & CSF leak & Full recovery \\
\hline & VA-BA (NA) & $\begin{array}{l}\mathrm{SAH} \text {, lethargic, acute } \\
\text { respiratory failure }\end{array}$ & $\begin{array}{l}\text { Transoral- } \\
\text { transclival }\end{array}$ & $\begin{array}{l}\text { Aneurysm } \\
\text { obliterated }\end{array}$ & $\begin{array}{l}\text { CSF leak, meningitis, } \\
\text { slip-out of the clip }\end{array}$ & Death \\
\hline \multirow[t]{2}{*}{$\begin{array}{l}\text { Hayakawa et al., } \\
1981\end{array}$} & $\begin{array}{l}\text { BA-AICA } \\
\text { (NA) }\end{array}$ & NA & $\begin{array}{l}\text { Transoral- } \\
\text { transclival }\end{array}$ & NA & None & Death \\
\hline & $\begin{array}{l}\text { BA-AICA } \\
\text { (NA) }\end{array}$ & NA & $\begin{array}{l}\text { Transoral- } \\
\text { transclival }\end{array}$ & NA & Meningitis, hydrocephalus & Full recovery \\
\hline
\end{tabular}


Table 1. continued.

\begin{tabular}{|c|c|c|c|c|c|c|}
\hline Authors \& Year & $\begin{array}{l}\text { Aneurysm } \\
\text { location } 8 \\
\text { size }(\mathbf{m m})\end{array}$ & $\begin{array}{l}\text { Clinical } \\
\text { presentation }\end{array}$ & Approach & $\begin{array}{l}\text { Post-operative } \\
\text { imaging }\end{array}$ & Complications & Outcome \\
\hline $\begin{array}{l}\text { Hayakawa et al., } \\
1981\end{array}$ & $\begin{array}{l}\text { BA-AICA } \\
\text { (NA) }\end{array}$ & NA & $\begin{array}{l}\text { Transoral- } \\
\text { transclival }\end{array}$ & NA & Hydrocephalus & Full recovery \\
\hline \multirow[t]{2}{*}{$\begin{array}{l}\text { Matricali et al., } \\
1981\end{array}$} & $\mathrm{BA}(\mathrm{NA})$ & $\mathrm{SAH}$, headache & $\begin{array}{l}\text { Transoral- } \\
\text { transclival }\end{array}$ & $\begin{array}{l}\text { Incomplete } \\
\text { closure of the } \\
\text { aneurysm }\end{array}$ & CSF leak & Death \\
\hline & $\mathrm{BA}(\mathrm{NA})$ & $\mathrm{SAH}$, headache & $\begin{array}{l}\text { Transoral- } \\
\text { transclival }\end{array}$ & NA & None & Full recovery \\
\hline $\begin{array}{l}\text { Litvak et al., } \\
1981\end{array}$ & $\mathrm{BA}(\mathrm{NA})$ & Syncope, seizure, come & $\begin{array}{l}\text { Transoral- } \\
\text { transclival }\end{array}$ & $\begin{array}{l}\text { Slip-out of the } \\
\text { clip }\end{array}$ & Slip-out of the clip & Full recovery \\
\hline $\begin{array}{l}\text { Hitchcock et al., } \\
1983\end{array}$ & VA (NA) & $\begin{array}{l}\text { Collapse, loss of } \\
\text { consciousness }\end{array}$ & $\begin{array}{l}\text { Transoral-trans- } \\
\text { clival }\end{array}$ & $\begin{array}{l}\text { Aneurysm } \\
\text { obliterated }\end{array}$ & CSF leak, meningitis & Full recovery \\
\hline \multirow[t]{3}{*}{$\begin{array}{l}\text { Archer et al., } \\
1987\end{array}$} & $\mathrm{BA}(\mathrm{NA})$ & SAH, coma & $\begin{array}{l}\text { Transoral- } \\
\text { transclival + Le } \\
\text { Fort I }\end{array}$ & NA & None & Full recovery \\
\hline & $\mathrm{BA}(\mathrm{NA})$ & $\begin{array}{l}\text { HH grade I, headache, } \\
\text { neck stiffness }\end{array}$ & $\begin{array}{l}\text { Transoral- } \\
\text { transclival } \\
\text { + Le Fort I }\end{array}$ & NA & $\begin{array}{l}\text { Pharyngeal wound } \\
\text { infection }\end{array}$ & Full recovery \\
\hline & $\mathrm{BA}(\mathrm{NA})$ & $\mathrm{HH}$ grade I, SAH & $\begin{array}{l}\text { Transoral- } \\
\text { transclival } \\
+ \text { Le Fort I }\end{array}$ & NA & None & Full recovery \\
\hline $\begin{array}{l}\text { Yamashita et al., } \\
1989\end{array}$ & VA-PICA (5) & SAH & $\begin{array}{l}\text { Transoral- } \\
\text { transclival }\end{array}$ & NA & Hemiparesis, meningitis & Full recovery \\
\hline $\begin{array}{l}\text { Crockard et al., } \\
1991\end{array}$ & $\begin{array}{l}\text { BA-AICA } \\
\text { (NA) }\end{array}$ & $\begin{array}{l}\text { SAH, trochlear and abdu- } \\
\text { cent nerves palsies }\end{array}$ & $\begin{array}{l}\text { Transoral- } \\
\text { transclival } \\
+ \text { Le Fort I }\end{array}$ & $\begin{array}{l}\text { Aneurysm } \\
\text { obliterated }\end{array}$ & None & Full recovery \\
\hline $\begin{array}{l}\text { de Los Reyes et al., } \\
1992\end{array}$ & $\mathrm{BA}(\mathrm{NA})$ & Headache & $\begin{array}{l}\text { Transoral- } \\
\text { transclival } \\
+ \text { Le Fort I }\end{array}$ & $\begin{array}{l}\text { Aneurysm } \\
\text { obliterated }\end{array}$ & CSF leak & Full recovery \\
\hline \multirow[t]{5}{*}{$\begin{array}{l}\text { Ogilvy et al., } \\
1996\end{array}$} & $\mathrm{BA}(\mathrm{NA})$ & $\mathrm{HH}$ grade I, SAH & $\begin{array}{l}\text { Transfacial- } \\
\text { transclival }\end{array}$ & $\begin{array}{l}\text { Aneurysm } \\
\text { obliterated }\end{array}$ & None & Full recovery \\
\hline & AICA (NA) & $\mathrm{HH}$ grade III, SAH & $\begin{array}{l}\text { Transfacial- } \\
\text { transclival }\end{array}$ & $\begin{array}{l}\text { Aneurysm } \\
\text { obliterated }\end{array}$ & SAH, CSF leak & Death \\
\hline & PICA (NA) & $\begin{array}{l}\text { HH grade II, bilateral } \\
\text { abducent nerve palsy }\end{array}$ & $\begin{array}{l}\text { Transfacial- } \\
\text { transclival }\end{array}$ & $\begin{array}{l}\text { Aneurysm } \\
\text { obliterated }\end{array}$ & $\begin{array}{l}\text { CSF leak (other } 2 \text { pz had } \\
\text { CSF leak) }\end{array}$ & Full recovery \\
\hline & VA-BA (NA) & $\begin{array}{l}\text { HH grade III, abducent } \\
\text { nerve palsy }\end{array}$ & $\begin{array}{l}\text { Transfacial- } \\
\text { transclival }\end{array}$ & $\begin{array}{l}\text { Aneurysm } \\
\text { obliterated }\end{array}$ & $\begin{array}{l}\text { Delayed trochlear nerve } \\
\text { palsy }\end{array}$ & $\begin{array}{l}\text { Cranial Nerve } \\
\text { Lesion }\end{array}$ \\
\hline & $\begin{array}{l}\text { BA (NA) + } \\
\text { fusiform dila- } \\
\text { tation }\end{array}$ & $\begin{array}{l}\mathrm{HH} \text { grade II, abducent } \\
\text { nerve palsy }\end{array}$ & $\begin{array}{l}\text { Transfacial- } \\
\text { transclival }\end{array}$ & $\begin{array}{l}\text { Aneurysm } \\
\text { obliterated }\end{array}$ & None, CSF leak & Full recovery \\
\hline $\begin{array}{l}\text { Stacey et al., } \\
2000\end{array}$ & $\begin{array}{l}\text { BA-AICA } \\
\text { (NA) }\end{array}$ & NA & $\begin{array}{l}\text { Transoral- } \\
\text { transclival }\end{array}$ & NA & NA & NA \\
\hline $\begin{array}{l}\text { Imamura et al., } \\
2001\end{array}$ & VA-PICA (3) & $\begin{array}{l}\mathrm{HH} \text { grade II, SAH, } \\
\text { headache }\end{array}$ & $\begin{array}{l}\text { Transoral- } \\
\text { transclival }\end{array}$ & $\begin{array}{l}\text { Aneurysm } \\
\text { obliterated }\end{array}$ & None & Full recovery \\
\hline
\end{tabular}

HH Hunt and Hess grade, NA not available, SAH subarachnoid hemorrhage, CSF cerebrospinal fluid, OTR osteotendinous reflexes, BA basilar artery, VA vertebral artery, AICA anteriorinferior cerebellar artery, PICA posteriorinferior cerebellar artery, pz patient, * computerized tomography (CT) and/or magnetic resonance imaging (MRI) and/or arteriography 
of 9 aneurysms were clipped via the nose.

In particular, as shown in Figure 4 (an anatomical and schematic picture demonstrating different vascular areas reached via extended endoscopic endonasal approaches until now; Laboratory of Surgical NeuroAnatomy of Barcelona), the transtuberculum/transplanum approach allows the exposure of the pre-communicating and post-communicating segments of the anterior cerebral arteries ( $\mathrm{A} 1$ and $\mathrm{A} 2$ ), the anterior communicating artery (AcomA), the frontopolar arteries (FPA), the superior hypophyseal arteries (sha), the proximal segment of the ophthalmic arteries (OphA) and the supraclinoid portion of the internal carotid arteries (ICA); the sellar/parasellar approach provides an optimal visualization of the cavernous portion of the ICA and superior hypophyseal arteries (sha). The transclival approach allows the exposure of the basilar artery (BA), the posterior cerebral arteries (PCA), the posterior communicating arteries (PcomA), the superior cerebellar arteries (SCA), the anterior inferior cerebellar arteries (AICA), the vertebral arteries (VA), the posterior inferior cerebellar arteries (PICA) and the anterior spinal artery (ASA) (Figures 4 and 5).

The first case of aneurysm clipping via the nose was published in 2006 by Kassam et al. ${ }^{(17)}$. It was a case of a large vertebral artery aneurysm that, after the endovascular trapping, was completely clipped by the endoscopic endonasal transclival approach. The dura was reconstructed with an intradural inlay Duragen graft (Integra Life Sciences, Boston, MA, USA), followed by an extradural onlay allograft plus fibrin glue and a Foley catheter balloon, inserted to provide support to the grafts and to prevent graft migration. Intranasal silastic splints were sutured in place bilaterally, and a lumbar drain was placed. The patient was discharged with an improvement of preoperative symptoms including weakness, incoordination and sensory changes. One year later, the same group reported a case of a 56-year-old woman found to have two unruptured aneurysms: an anterior communicating artery (AComA) aneurysm and a superior hypophyseal artery (sha) aneurysm ${ }^{(18)}$. The aneurysm was clipped by an endoscopic endonasal transtuberculum/transplanum approach and, except from a mild postoperative confusion, the patient was free of any infection and/or CSF leak.

In 2007, Kitano et al. (44) reported a case of a small incidental unruptured AComA aneurysm, which was clipped via a microscopic endoscopic-assisted sublabial-transtuberculum/transplanum approach, after a subtotal removal of a macroadenoma. An autologous fascia graft was placed subdurally on the anterior cranial base to cover the dural opening and the fascial graft was sutured to the dural edges. Moreover, ceramic cement was applied to reconstruct the bony defect, reinforce the dural closure, and inhibit CSF leakage. Interestingly, Kitano et al. performed an anatomical dissection of two cadaveric heads to confirm the usefulness of the extended endoscopic endonasal approach to expose the AcomA complex and its lesions. In the same year, Eloy et al. ${ }^{(45)}$ used a Weck clip (Weck Closure Systems Research, Triangle Park, NC, USA), which provided a low profile for closure, to obliterate an aneurysm of the mid-basilar trunk via a microscopic sublabial-transclival approach. For persistent fusiform dilation in the region of the aneurysm, endovascular stenting was also performed and, at the 3-month follow-up examination, the patient had made a full recovery. Moreover, Acerbi et al. (46) demonstrated the use of nitinol U-Clips to reconstruct the cranial base dura in a series of 11 patients that included 1 case with a mid-basilar trunk aneurysm. One year later, Enseñat et al. ${ }^{(42)}$ managed a ruptured vertebral-posterior inferior cerebellar artery complex (VA-PICA) aneurysm, successfully treated via an endoscopic endonasal extended approach to the clival region. It was the first report of a pure endoscopically treated VA-PICA aneurysm. The aneurysm was accurately reached and successfully clipped from the parent artery. For reconstruction of the skull base defect a copolymer of I-lactic acid and glycolic acid (LactoSorb) as a bone substitute was used and, as a dural substitute, fascia lata. In detail, an inlay fascia lata graft was placed intradurally, and another large piece of the same material exceeding the size of the osseous defect was placed over it; then, a fragment of LactoSorb was wedged into the extradural space. Finally, it was placed as a Hadad-Bassagaisteguy Flap (HBF) (47), fixed with an oxidized cellulose polymer and fibrin glue. A postoperative arteriogram showed proper placement of the clip, obliteration of the aneurysm, and the patency of the VA and the PICA. Seven days after surgery, the patient had a CSF rhinorrhea and underwent endoscopic endonasal repair of a CSF leak. The patient was discharged 1 month after reoperation without any neurological deficit.

In the same year, Froelich et al. ${ }^{(40)}$ and Germanwala et al. ${ }^{(48)}$ performed an endoscopic endonasal transtuberculum/transplanum surgery for aneurysms of the anterior circulation. A multilayer reconstruction technique was used in all cases and the postoperative course was uneventful.

The last report appeared in the literature in 2012 by Drazin et al. ${ }^{(49)}$. The authors used an expanded endoscopic endonasaltransclival approach to successfully clip a basilar trunk aneurysm and feeding arteriovenous malformation (AVM) vessel. In this case, a small straight Yaşargil mini aneurysm clip (Aesculap AG, Tuttlingen, Germany) was applied across the aneurysm using an endonasal clip applier (Sephernia Neurosurgical micro-instruments, Karl Storz, Tuttlingen, Germany) and the dural defect was repaired using a multilayer technique, with placement of the nasoseptal flap. The patient was discharged from the hospital without any neurological deficits.

\section{Results}

Forty-eight cases, mostly case reports, were included and carefully analyzed. Data about these papers are summarized in Table 
1 and Table 2. Only abstracts and/or full text manuscripts in English were analyzed, with the aim of calculating complication and surgical success rates for both "traditional" ventral (transcervical-transclival, transoral-transclival, transfacial-transclival) and extended transsphenoidal (microscopic endoscopic-assisted sublabial-transtuberculum/transplanum, microscopic sublabialtransclival, extended endoscopic endonasal) approaches. As shown in Figure 6, the overall complication rate for traditional ventral approaches, namely the transcervical-transclival, the transoral-transclival and the transfacial-transclival was $74 \%$ (26/35), whereas for extended transsphenoidal approaches, i.e. the microscopic endoscopic-assisted sublabial-transtuberculum/transplanum, the microscopic sublabial-transclival, the endoscopic endonasal transclival and the endoscopic endonasal transtuberculum/transplanum, the rate was found to be $44 \%$ (4/9).

The overall surgical success rate for traditional ventral approaches was found to be $87 \%(13 / 15)$, while for an extended transsphenoidal surgery, it was $78 \%$ (7/9).

\section{Discussion}

The management of both ruptured and unruptured cerebral aneurysms has undergone significant evolution in the modern era. Nowadays, it can be considered as the result of a close cooperation between different specialists, i.e. the ENT surgeon, the neuroradiologist, the neurologist and the neurosurgeon ${ }^{(50)}$. In such a combined neurovascular team, each member plays a well-defined role, offering his contribution to the final result, specifically tailored to a single patient. During the last decades, the endovascular technique has rapidly evolved thanks to the Food and Drug Administration approval of Guglielmi detachable coils in 1995, which since then allowed successful treatment of a great deal of cerebral aneurysms in many centers all over the world. In the latter half of the 1990s, as experience of endovascular techniques spread, such treatment began to displace open surgery and the International Subarachnoid Aneurysm Trial (ISAT) was set up to compare the efficacy of the two treatments (51,52). The endovascular technique has changed the way we practice neurosurgery. Endovascular therapy has largely replaced microsurgery as the first line treatment modality for the majority of cerebral aneurysms. Recently, reconstructive endovascular treatment, including stent, stent-assisted coiling or flow diverters, has been applied to complex posterior circulation aneurysms and high viscosity liquid embolization agents have been used effectively, particularly in the treatment of distal posterior circulation aneurysms ${ }^{(53,54)}$.

The history of intracranial surgery for aneurysms is not a long one. The first direct operation on an intracranial aneurysm was performed by Dott, who wrapped a ruptured aneurysm in $1933^{(55)}$ and the first obliterative clipping of an aneurysm was performed by Dandy in $1938^{(56)}$. The results of surgery improved dramatically when the operating microscope was introduced in the 1960s and its propagation in the 1970s, 1980s and 1990s greatly influenced the results of aneurysm surgery. The pterional approach first described by Yaşargil in those years, allowed an excellent exposure of the circle of Willis and the management of aneurysm affecting this anatomical region ${ }^{(11,57)}$. Since then, important intraoperative adjuncts such as micro-Doppler ultrasonography (MDU), intraoperative angiography (IOA), and near-infrared indocyanine green (ICG) video angiography have been emerging as very useful tools in vascular surgery. However, during the endovascular era, the challenge for contemporary vascular neurosurgeons is to understand the different but complementary role each treatment modality currently has to offer, and to maintain the proficiency and technical skills to deal with an emergence of complex and in many cases, recurrence of previously coiled aneurysms.

Despite these great advances made both in surgical and endovascular techniques, the complexity of some vascular lesions makes their treatment still a challenge for vascular teams, as they are associated with a high incidence of complications, which is particularly true for posterior circulating aneurysms. Indeed, reviewing the literature, it is apparent that posterior circulation aneurysms present as significant endovascular challenge to the neurointerventionalist as it does to the neurosurgeon. Historically, such vascular lesions present a difficult challenge because they are located in an exquisitely eloquent and sometimes difficult-to-reach area in the posterior cranial fossa.

For such reasons starting from the late 1960's, the ventral route has been advocated as a valid alternative to reach those lesions. The ventral pathway was considered a reasonable option because it permits direct access to the surgical field obviating brain retraction and obtaining an early and safe proximal and distal vascular control. Since then, more than thirty authors report the use of transcervical-transclival, transoral-transclival, transfacialtransclival and, finally, extended transsphenoidal approaches to occlude posterior circulating aneurysms. As demonstrated by the publication of more than 45 cases in the last 50 years, the ventral pathway is an attractive and valid alternative to established neurosurgical procedures currently in use for the treatment of intracranial aneurysms.

Nowadays, the oribitozygomatic, transpetrous, retrosigmoid, or far-lateral approaches have been well recognized as preferred pathways to treat complex posterior circulation aneurysms that cannot be treated by endovascular therapy. On the other hand, tranditional ventral approaches including transcervical-transclival, transoral-transclival, and transfacial-transclival routes have been gradually discharged and, actually, they can be considered seldom. 
Table 2. Clinical and operative characteristics in 9 reported patients with intracranial aneurysms treated via extended transsphenoidal approaches (microscopic endoscopic-assisted sublabial-transtuberculum/transplanum, microscopic sublabial-transclival, extended endoscopic endonasal), literature review.

\begin{tabular}{|c|c|c|c|c|c|c|}
\hline Authors \& Year & $\begin{array}{l}\text { Aneurysm } \\
\text { location } \& \\
\text { size }(\mathbf{m m})\end{array}$ & $\begin{array}{l}\text { Clinical } \\
\text { presentation }\end{array}$ & Approach & $\begin{array}{l}\text { Post-operative } \\
\text { imaging }\end{array}$ & Complications & Outcome \\
\hline $\begin{array}{l}\text { Kassam et al., } \\
2006\end{array}$ & VA (11) & $\begin{array}{l}\text { Left leg weakness and } \\
\text { sensory changes }\end{array}$ & $\begin{array}{l}\text { Endoscopic endo- } \\
\text { nasal-transclival }\end{array}$ & Aneurysm obliterated & None & Full recovery \\
\hline $\begin{array}{l}\text { Kassam et al., } \\
2007\end{array}$ & Sha (5) & Incidental & $\begin{array}{l}\text { Endoscopic } \\
\text { endonasal } \\
\text { transtuberculum/ } \\
\text { transplanum }\end{array}$ & Aneurysm obliterated & $\begin{array}{l}\text { Mild postoperative } \\
\text { confusion }\end{array}$ & Full recovery \\
\hline $\begin{array}{l}\text { Kitano et al., } \\
2007\end{array}$ & AComA (NA) & Incidental & $\begin{array}{l}\text { Microscopic } \\
\text { endoscopic-as- } \\
\text { sisted sublabial- } \\
\text { transtuberculum/ } \\
\text { transplanum }\end{array}$ & Aneurysm obliterated & None & Full recovery \\
\hline $\begin{array}{l}\text { Eloy et al., } \\
2007\end{array}$ & $\mathrm{BA}(2.5)$ & $\mathrm{HH}$ grade III & $\begin{array}{l}\text { Microscopic subla- } \\
\text { bial-transclival }\end{array}$ & $\begin{array}{l}\text { Persistent fusiform } \\
\text { dilation of } \mathrm{BA}\end{array}$ & $\begin{array}{l}\text { Vasospasm, } \\
\text { hydrocephalus }\end{array}$ & Full recovery \\
\hline $\begin{array}{l}\text { Acerbi et al., } \\
2010\end{array}$ & $\begin{array}{l}\mathrm{BA}(\mathrm{NA})+ \\
\text { fusiform dila- } \\
\text { tation }\end{array}$ & SAH & $\begin{array}{l}\text { Microscopic subla- } \\
\text { bial-transclival }\end{array}$ & $\begin{array}{l}\text { Aneurysm obliterated, } \\
\text { reduced fusiform } \\
\text { dilation }\end{array}$ & $\begin{array}{l}\text { Vasospasm, DVT right } \\
\text { leg, hydrocephalus }\end{array}$ & Full recovery \\
\hline $\begin{array}{l}\text { Ensenat et al., } \\
2011\end{array}$ & VA-PICA (1.2) & $\begin{array}{l}\text { Headache and decreased } \\
\text { level of consciousness }\end{array}$ & $\begin{array}{l}\text { Endoscopic endo- } \\
\text { nasal-transclival }\end{array}$ & Aneurysm obliterated & $\begin{array}{l}\text { CSF leak, } \\
\text { hydrocephalus }\end{array}$ & Full recovery \\
\hline $\begin{array}{l}\text { Froelich et al., } \\
2011\end{array}$ & AComA (7) & Incidental & $\begin{array}{l}\text { Endoscopic } \\
\text { endonasal } \\
\text { transtuberculum/ } \\
\text { transplanum }\end{array}$ & Aneurysm obliterated & None & Full recovery \\
\hline $\begin{array}{l}\text { Germanwala et al., } \\
2011\end{array}$ & $\begin{array}{l}\text { Ophtalmic } \\
\text { (5), Paracli- } \\
\text { noid (10) }\end{array}$ & $\mathrm{HH}$ grade II & $\begin{array}{l}\text { Endoscopic } \\
\text { endonasal } \\
\text { transtuberculum/ } \\
\text { transplanum }\end{array}$ & Aneurysm obliterated & None & Full recovery \\
\hline $\begin{array}{l}\text { Drazin et al., } \\
2012\end{array}$ & $\begin{array}{l}\text { BA (4), cere- } \\
\text { bellar AVM }\end{array}$ & $\mathrm{HH}$ grade II & $\begin{array}{l}\text { Endoscopic endo- } \\
\text { nasal-transclival }\end{array}$ & $\begin{array}{l}\text { Partial aneurysm oc- } \\
\text { clusion (1st surgery) }\end{array}$ & None & Full recovery \\
\hline
\end{tabular}

HH Hunt and Hess grade, NA not available, SAH subarachnoid hemorrhage, CSF cerebrospinal fluid, DVT deep venous thrombosis, AComA anterior communicating artery, Sha superior hypophyseal artery, BA basilar artery, VA vertebral artery, PICA posteriorinferior cerebellar artery, AVM arteriovenous malformation, * computerized tomography $(\mathrm{CT})$ and/or magnetic resonance imaging (MRI) and/or arteriography.

However, in recent years, thanks to Kassam et al. ${ }^{(17)}$, some reports have been published stating that specific midline anterior and posterior circulating aneurysms not amenable to endovascular treatment could be managed via the anterior route, in particular throughout the extended endoscopic endonasal route. The evolution of the transsphenoidal technique, which was initially reserved to sellar lesions, has lead in the last decades to a progressive possibility to access the different areas of the skull base. Indeed, such a route allows midline access and visibility to the suprasellar, retrosellar, parasellar and clival space while obviating brain retraction, and makes it possible to treat transsphenoidally a variety of midline skull base lesions. Particularly, among transsphenoidal surgeries, the endoscopic endonasal pathway allows the treatment of wide range of the midline skull base region pathologies, with the advantage of a wider vision of the surgical field, less traumatism of the brain structures, lower cases of cranial nerves lesions, greater facility in the treatment of possible recurrences and reduced complications.

Applied to vascular surgery, the extended endoscopic endonasal approach offers some advantages due to the properties of the endoscope itself; it provides a wider, close-up view of the surgical field thus allowing, for selected midline vascular lesions, the achievement of a safe proximal and distal vascular control in 
the majority of cases, a comfortably bimanual dissection and a close and detailed visualization of the main neurovascular structures, i.e. small perforator arteries without any brain retraction (58-60).

The endonasal route provides different advantages compared to previous approaches used. In general, it is less traumatic, as compared with the transoral pathway, and the bacterial flora of the nose is less virulent and represented than that of the oral cavity, thus reducing the risk of infection of the CSF. Moreover, the availability of broad-spectrum antibiotics with adequate CSF penetration, such as third-generation cephalosporin, allows patients to recover without complications despite clinical evidence of meningitis ${ }^{(39,61)}$. Very relevant is that the time to reach the lesion is mainly focussed on the extradural space, i.e. nose or sphenoid cavity, while during classical neurosurgical approaches the main part of the procedure is aimed to reach the deep vascular territory by shifting vital neurovascular structures. Bleeding control can be seen as one of the major issues during intracranial aneurysm surgery. This is particularly challenging for endonasal approaches in which blood can obscure the main anatomical landmarks of the surgical field and dirties the endoscope lens, causing greater difficulty with visualization. Another important point during this kind of surgery is the impossibility to perform vascular by-passes and the difficulties that may be encountered for the application of proximal and distal temporary clips coupled with the room for a permanent clip. On the other hand, the development of new techniques in skull base surgery also allowed maintaining, in most cases, the same basic principles of the microsurgical technique for vascular surgery. Indeed, the endoscopic endonasal technique in selected cases can allow the achievement of a safe proximal and distal vascular control, a comfortably bimanual dissection, and a close and detailed visualization of the main neurovascular structures (especially small perforator arteries). For such reason, we consider that the same principles of microsurgical vascular repair must be strictly followed during transsphenoidal surgery.

Regarding surgical success and overall complications, as shown in the present paper, the overall surgical success seems to be comparable between "traditional" ventral approaches and extended transsphenoidal routes ( $87 \%$ vs. $78 \%$ ). On the other hand, the overall complication rate using transsphenoidal approaches, such as the microscopic endoscopic-assisted sublabial-transtuberculum/transplanum, the microscopic sublabial-transclival, the endoscopic endonasal transclival and the endoscopic endonasal transtuberculum/transplanum, has been dramatically reduced compared to previous approaches used (44\% vs. $74 \%)$.

However, the transsphenoidal surgery and, as a specific reference, endoscopic endonasal surgery, brings some disadvantages that should be taken in consideration. The exposure provided by the approach is limited to certain anatomical skull base regions, proximal and distal vascular control may be hard in case of difficulties. Furthermore, the inability to perform a bypass graft, risk of CSF leak and the significant endoscopic skills required are other drawbacks, which could make the ventral corridor indicated only in very selected cases. Moreover, in the endonasal approach the surgical corridor is narrow, with less leaving room for the surgeon to comfortably dissect and definitively clip the aneurysm.

Finally, regarding the issue of transsphenoidal surgery applied to cerebrovascular diseases, it should be stressed that, at present, this kind of approach is still in its infancy - only 9 cases in the current literature - so we have to wait further developments, in terms of surgical technique as well as tools and reconstruction materials, to reach proper conclusions. Moreover, these are the reasons why it is not possible at present to make a comparison with the relevant open and endovascular pathways.

Another major issue emerging in recent years refers to skull base reconstruction, which continues to be a major challenge and an obstacle for the use and acceptance of the expanded endonasal approaches ${ }^{(62,63)}$. A significant progress in reconstruction of dural defects has been made thanks to the description of the Hadad-Bassagasteguy flap (HBF) in $2006^{(47)}$. The HBF is a witness reconstructive technique for extensive defects of the anterior, middle, clival, and parasellar skull base and its use has resulted in a sharp decrease in the incidence of postoperative CSF leaks after endoscopic endonasal approaches.

\section{Conclusion}

Increasing technological developments have led to the application of the endoscope to cranial base and, more recently, cerebrovascular surgery. However, no doubt, aneurysm surgery is greatly advanced with the endovascular techniques and room for surgery has been and will be progressively reduced. We stress that the present and future of cerebral aneurysm treatment is the endovascular technique. With this in mind, our paper wants to be a reconnaissance of what has been done via "the anterior route" and a notification of the existence of this "surgical window", which should be sparingly used. Just a few selected cases in specialized centers, where transsphenoidal approaches are routinely performed, may be treated with such techniques alone or in combination with the other procedures.

\section{Authorship contribution}

ADS: Design, acquisition of data, analysis and interpretation of data, drafting of the manuscript, final approval of draft. MdN: Design, acquisition of data, analysis and interpretation of data, drafting and revision of the manuscript, final approval of draft JE: Analysis and interpretation of data, revision of the manus- 
cript, final approval of draft. IA: Analysis and interpretation of data, revision of the manuscript, final approval of draft. MB-S: Analysis and interpretation of data, revision of the manuscript, final approval of draft. LMC: Analysis and interpretation of data, revision of the manuscript, final approval of draft. AP-G: Design, Analysis and interpretation of data, revision of the manuscript, final approval of draft. PC: Analysis and interpretation of data, revision of the manuscript, final approval of draft.

\section{Acknowlegdement}

The present paper has been partially supported by the Marató TV3 Grant Project: [411/U/2011 - TITLE: Quantitative analysis and computer aided simulation of minimally invasive approaches for intracranial vascular lesions.]

\section{Conflicts of Interest}

The authors have no personal financial or institutional interest in the devices described in this article.

\section{References}

1. Sano K, Jinbo M, Saito I. [Vertebro-basilar aneurysms, with special reference to the transpharyngeal approach to basilar artery aneurysm]. No To Shinkei. 1966; 18: $1197-$ 1203.

2. Drake CG. Bleeding aneurysms of the basilar artery. Direct surgical management in four cases. J Neurosurg. 1961; 18: 230-238.

3. Jamieson KG. Aneurysms of the Vertebrobasilar System; Surgical Intervention in 19 Cases. J Neurosurg. 1964; 21: 781-797.

4. Day JD, Fukushima T, Giannotta SL. Cranial base approaches to posterior circulation aneurysms. J Neurosurg. 1997; 87: 544-554.

5. Gonzalez LF, Amin-Hanjani S, Bambakidis NC, Spetzler RF. Skull base approaches to the basilar artery. Neurosurg Focus. 2005; 19: E3.

6. Le Roux PD, Winn HR. Surgical approaches to basilar bifurcation aneurysms. Neurosurg Clin N Am. 1998; 9: 835-849.

7. Lister JR, Rhoton AL, Jr., Matsushima T, Peace DA. Microsurgical anatomy of the posterior inferior cerebellar artery. Neurosurgery. 1982; 10: 170-199.

8. Fox JL. Obliteration of midline vertebral artery aneurysm via basilar craniectomy. J Neurosurg. 1967; 26: 406-412.

9. Saito I, Takahashi H, Joshita H, et al. Clipping of vertebro-basilar aneurysms by the transoral transclival approach. Neurol Med Chir (Tokyo). 1980; 20: 753-758.

10. Wissinger JP, Danoff D, Wisiol ES, French LA. Repair of an aneurysm of the basilar artery by a transclival approach. Case report. J Neurosurg. 1967; 26: 417-419.

11. Yașargil MG. Microsurgery applied to neurosurgery. Stuttgart: George Thieme Verlag; 1969.

12. Drake CG. The surgical treatment of vertebral-basilar aneurysms. Clin Neurosurg. 1969; 16: 114-169.

13. Chou SN, Ortiz-Suarez HJ. Surgical treatment of arterial aneurysms of the vertebrobasilar circulation. J Neurosurg. 1974; 41: 671-680.

14. Alobid I, Ensenat J, Marino-Sanchez F, et al. Impairment of olfaction and mucociliary clearance after expanded endonasal approach using vascularized septal flap reconstruction for skull base tumors.
Neurosurgery. 2013; 72: 540-546.

15. Ensenat J, de Notaris $M$, Sanchez $M$, et al. Endoscopic endonasal surgery for skull base tumours: technique and preliminary results in a consecutive case series report. Rhinology. 2013; 51: 37-46.

16. Alobid I, Ensenat J, Marino-Sanchez F, et al. Expanded endonasal approach using vascularized septal flap reconstruction for skull base tumors has a negative impact on sinonasal symptoms and quality of life. Am J Rhinol Allergy. 2013; 27: 426-431

17. Kassam AB, Mintz AH, Gardner PA, et al. The expanded endonasal approach for an endoscopic transnasal clipping and aneurysmorrhaphy of a large vertebral artery aneurysm: technical case report. Neurosurgery. 2006; 59 (1Suppl 1): ONSE162-165.

18. Kassam AB, Gardner PA, Mintz A, et al. Endoscopic endonasal clipping of an unsecured superior hypophyseal artery aneurysm. Technical note. J Neurosurg. 2007; 107: 1047-1052.

19. Crockard HA, Koksel T, Watkin N. Transoral transclival clipping of anterior inferior cerebellar artery aneurysm using new rotating applier. Technical note. J Neurosurg. 1991; 75: 483-485.

20. Haselden FG, Brice JG. Transoral clivectomy; (a case report). J Maxillofac Surg. 1978; 6: 32-34.

21. Laine E, Allegre GE, Goutelle A, et al. [Vertebrobasilar aneurysms: selection of approach. Problem of peroperative adjuvants]. Neurochirurgie. 1972; 18: 317-332.

22. Laine $E$, Jomin M. [Indications and possibilities of the trans-bucco-pharyngeal approach in presence of an aneurysm of the vertebro-basilar confluence]. Neurochirurgie. 1977; 23: 307-314

23. Yamaura A, Makino $H$, Isobe $K$, et al. Repair of cerebrospinal fluid fistula following transoral transclival approach to a basilar aneurysm. Technical note. J Neurosurg. 1979; 50: 834-838.

24. Hitchcock E, Cowie R. Transoral-transclival clipping of a midline vertebral artery aneurysm. J Neurol Neurosurg Psychiatry. 1983; 46: 446-448.

25. Litvak J, Sumners TC, Barron JL, Fisher LS. A successful approach to vertebrobasilar aneurysms. Technical note. J Neurosurg. 1981; 55: 491-494.
26. Imamura J, Ikeyama Y, Tsutida E, Moroi J. Transoral transclival approach for intradural lesions using a protective bone baffle to block cerebrospinal fluid pulse energy--two case reports. Neurol Med Chir (Tokyo). 2001; 41: 222-226.

27. Yamashita K, Abe H, Ikota T, Tashiro $T$, Kamiyama H. [Transoral transclival approach to vertebral-posterior inferior cerebellar artery aneurysm. Case report]. Neurol Med Chir (Tokyo). 1989; 29: 342-346.

28. Stacey RJ, Wadley JP, Kitchen N. Imageguided transoral clipping of basilar aneurysm. Br J Neurosurg. 2000; 14: 593-594.

29. Drake CG. Treatment of aneurysms of the posterior cranial fossa. In: Krayenbuehl H, Maspes PE, Sweet WH, editors. Progress in Neurological Surgery Microsurgical Approach to Cerebro-Spinal Lesion. 9. Basel, Miinchen, Paris, London, New York, Sidney: S Karger AG; 1978. p. 122-194.

30. Hashi K, Hakuba A, Ikuno H, Nishimura S. [A midline vertebral artery aneurysm operated via transoral transclival approach (author's transl)]. No Shinkei Geka. 1976; 4: 183-189.

31. Matricali B, van Dulken H. Aneurysm of fenestrated basilar artery. Surg Neurol. 1981; 15: 189-191.

32. Hayakawa T, Kamikawa K, Ohnishi T, Yoshimine T. Prevention of postoperative complications after a transoral transclival approach to basilar aneurysms. Technical note. J Neurosurg. 1981; 54: 699-703.

33. Hayakawa T, Kamikawa K, Ohnishi T, Yoshimine T. [Transoral transclival approach to aneurysms of the basilar artery - experience with three cases (author's transl)]. Neurol Med Chir (Tokyo). 1981; 21: 477-484.

34. Hayakawa T, Yamada K, Yoshimine T. [Transoral transclival approach: anatomical and technical notes]. No Shinkei Geka. 1989; 17: 609-614.

35. Archer DJ, Young S, Uttley D. Basilar aneurysms: a new transclival approach via maxillotomy. J Neurosurg. 1987; 67: 54-58.

36. de los Reyes RA, Kantrowitz AB, Detwiler PW, et al. Transoral-transclival clipping of a giant lower basilar artery aneurysm. Surg Neurol. 1992; 38: 379-382.

37. Crockard HA. The transoral approach to the base of the brain and upper cervical cord. Ann R Coll Surg Engl. 1985; 67: 321-325.

38. Crockard H, Sen C. The transoral approach 
for the management of intradural lesions at the craniovertebral junction: review of 7 cases. Neurosurgery. 1991; 28: 88-98.

39. Ogilvy CS, Barker FG, 2nd, Joseph MP et al. Transfacial transclival approach for midline posterior circulation aneurysms. Neurosurgery. 1996; 39: 736-742.

40. Froelich S, Cebula H, Debry C, Boyer P. Anterior communicating artery aneurysm clipped through an endoscopic endonasal approach: Technical note. Neurosurgery. 2011. 68; (2 Suppl): 310-316

41. Lai LT, Morgan MK, Chin DC, et al. A cadaveric study of the endoscopic endonasal transclival approach to the basilar artery. J Clin Neurosci. 2013; 20: 587-592.

42. Enseñat J, Alobid I, de Notaris M, et al. Endoscopic endonasal clipping of a ruptured vertebral-posterior inferior cerebellar artery aneurysm: technical case report. Neurosurgery. 2011; 69 (1 Suppl): onsE121128.

43. Di Somma A, de Notaris M, Stagno V, et al. Extended endoscopic endonasal approaches for cerebral aneurysms: anatomical, virtual reality and morphometric study. BioMed Research International. 2014; (in press). http://www.hindawi.com/journals/bmri/ aip/703792/

44. Kitano M, Taneda M. Extended transsphenoidal approach to anterior communicating artery aneurysm: aneurysm incidentally identified during macroadenoma resection: technical case report. Neurosurgery. 2007; 61 (Suppl 2): E299-300.

45. Eloy JA, Carai A, Patel AB, Genden EM, Bederson JB. Combined endoscope-assisted transclival clipping and endovascular stenting of a basilar trunk aneurysm: case report. Neurosurgery. 2008; 62 (Suppl 1): 142-144.

46. Acerbi F, Genden E, Bederson J. Circumferential watertight dural repair using nitinol U-clips in expanded endonasal and sublabial approaches to the cranial base. Neurosurgery. 2010; 67 (Suppl Operative): 448-456.

47. Hadad G, Bassagasteguy L, Carrau RL, et al. A novel reconstructive technique after endoscopic expanded endonasal approaches: vascular pedicle nasoseptal flap. Laryngoscope. 2006; 116 (10): 1882-
1886

48. Germanwala AV, Zanation AM. Endoscopic endonasal approach for clipping of ruptured and unruptured paraclinoid cerebral aneurysms: case report. Neurosurgery. 2011; 68 (Suppl Operative): 234-249.

49. Drazin D, Zhuang L, Schievink WI, Mamelak AN. Expanded endonasal approach for the clipping of a ruptured basilar aneurysm and feeding artery to a cerebellar arteriovenous malformation. J Clin Neurosci. 2012; 19: 144-148.

50. Lund VJ, Stammberger $\mathrm{H}$, Nicolai $\mathrm{P}$, et al. European position paper on endoscopic management of tumours of the nose, paranasal sinuses and skull base. Rhinology Supplement. 2010 (22): 1-143.

51. Molyneux A, Kerr R, Stratton I, et al. International Subarachnoid Aneurysm Trial (ISAT) of neurosurgical clipping versus endovascular coiling in 2143 patients with ruptured intracranial aneurysms: a randomised trial. Lancet. 2002; 360: 1267-1274.

52. Molyneux AJ, Kerr RS, Yu LM, et al. International subarachnoid aneurysm trial (ISAT) of neurosurgical clipping versus endovascular coiling in 2143 patients with ruptured intracranial aneurysms: a randomised comparison of effects on survival, dependency, seizures, rebleeding, subgroups, and aneurysm occlusion. Lancet. 2005; 366: 809817.

53. Brinjikji W, Murad MH, Lanzino G, Cloft HJ, Kallmes DF. Endovascular treatment of intracranial aneurysms with flow diverters: a meta-analysis. Stroke. 2013; 44: 442-447.

54. Bogason ET, Patel AS, Cockroft KM Endovascular Embolization by Parent Artery Reconstruction of a Symptomatic Fusiform Posterior Cerebral Artery Aneurysm Using Onyx HD-500: A Neurointerventional Report. J Neuroimaging. 2013; 23: 518-522.

55. Dott N. Intracranial aneurysms. Cerebral arterio-radiography: Surgical treatment. Edin Med J. 1933; 1933: 219-234.

56. Dandy WE. Intracranial Aneurysm of the Internal Carotid Artery: Cured by Operation. Ann Surg. 1938; 107: 654-659.

57. Yașargil MG. Microneurosurgery. Stuttgart: George Thieme Verlag; 1984. 215-33 p.

58. Cappabianca P, Cavallo LM, de Divitiis E. Endoscopic endonasal transsphenoidal sur- gery. Neurosurgery. 2004; 55: 933-941.

59. de Divitiis E, Cappabianca P, Cavallo LM Endoscopic transsphenoidal approach: adaptability of the procedure to different sellar lesions. Neurosurgery. 2002; 51: 699707.

60. Spencer WR, Das K, Nwagu C, et al. Approaches to the sellar and parasellar region: anatomic comparison of the microscope versus endoscope. Laryngoscope. 1999; 109: 791-794.

61. Kono Y, Prevedello DM, Snyderman $\mathrm{CH}$, et al. One thousand endoscopic skull base surgical procedures demystifying the infection potential: incidence and description of postoperative meningitis and brain abscesses. Infect Control Hosp Epidemiol. 2011; 32: 77-83.

62. Mascarenhas L, Moshel YA, Bayad F, et al. The transplanum transtuberculum approaches for suprasellar and sellar-suprasellar lesions. Avoidance of CSF leak and lessons learned. World Neurosurg. 2013. pii: S1878-8750(13)00297-0.

63. Cappabianca P, Di Somma A, de Notaris M Rerum magistra experientia est: The evolution of modern endoscopic endonasal skull base surgery and reconstruction techniques. World Neurosurg. 2013. pii: S18788750(13)00557-3.

\section{Isam Alobid}

Rhinology Unit and Smell Clinic

Dept of Otorhinolaryngology

Hospital Clínic

Universitat de Barcelona

c/ Villarroel, 170

Barcelona 08036

Spain

TEL: +34-932-279 872

FAX: +34-932-275050

E-mail: isamalobid@gmail.com 